\title{
Cellulose acetate phthalate microencapsulation and delivery of plasmid DNA to the intestines
}

\begin{abstract}
Cellulose acetate phthalate (CAP) microcapsules were formulated to deliver plasmid DNA (pDNA) to the intestines. The microcapsules were characterized and were found to have an average diameter of $44.33 \pm 30.22 \mu \mathrm{m}$, and were observed to be spherical with smooth surface. The method to extract pDNA from CAP was modified to study the release profile of the pDNA. The encapsulated pDNA was found to be stable. Exposure to the acidic and basic pH conditions, which simulates the $\mathrm{pH}$ environment in the stomach and the intestines, showed that the release occurred in a stable manner in the former, whereas it was robust in the latter. The loading capacity and encapsulation efficiency of the microcapsules were low but the CAP recovery yield was high which indicates that the microcapsules were efficiently formed but the loading of pDNA can be improved. In vitro transfection study in 293FT cells showed that there was a significant percentage of green-fluorescent-protein-positive cells as a result of efficient transfection from CAP-encapsulated pDNA. Biodistribution studies in BALB/c mice indicate that DNA was released at the stomach and intestinal regions. CAP microcapsules loaded with pDNA, as described in this study, may be useful for potential gene delivery to the intestines for prophylactic or therapeutic measures for gastrointestinal diseases.
\end{abstract}

Keyword: Cellulose acetate phthalate; DNA delivery; Microencapsulation; Microparticles; Microsphere; Oral administration; Oral delivery; Plasmid DNA; Polymeric delivery system; Solvent evaporation 